\title{
Editorial
}

\section{TDP-43 Models Coverage}

\author{
Amber Dance, Alzheimer Research Forum
}

\section{MEET THE FIRST PUBLISHED TDP-43 MOUSE}

There is a new mouse in the laboratory for the study of amyotrophic lateral sclerosis (ALS). And there will soon be more: mice carrying a mutant version of human TAR DNA binding protein 43 (TDP-43) will likely herald a veritable herd of mice with various TDP-43 mutations. Robert Baloh and colleagues at Washington University in St. Louis, Missouri, reported on the first published transgenic model of TDP-43 proteinopathy, describing a mouse that shows features of both ALS and frontotemporal lobar degeneration with ubiquitin aggregates (FTLD-U), another TDP-43 proteinopathy that sometimes co-presents with ALS [1]. Surprisingly, the mouse lacks one common feature of human disease: although there are ubiquitinated protein inclusions in the animal's neurons, TDP-43 is not a component of the aggregates. This could indicate that TDP-43 aggregates are not central to disease - or simply suggest that the model is a poor facsimile of human disease.

John Trojanowski of the University of Pennsylvania in Philadelphia, who was not involved in the current research, applauded the publication. "This will energize the field," he said. Several laboratories are working on their own TDP-43 mouse models; Trojanowski joked good-naturedly that he was "crushed" to see Baloh's group make it into print publication first.

However, researchers warned against overinterpreting early results, particularly since the current publication lacked control mice overexpressing wild-type human TDP-43. "We have to be incredibly cautious as far as what these results mean," said Leonard Petrucelli of the Mayo Clinic in Jacksonville, Florida, who also was not a participant in the PNAS paper. "I think it is a bit of a stretch to say that it is an ALS model per se .... It begs additional work." Researchers should be particularly wary, he noted, given the ALS field's history with animal models. The mouse overexpressing human superoxide dismutase 1, long a standard in ALS laboratories, has frequently responded to drugs that later failed in people.

Baloh, first author Iga Wegorzewska, and colleagues constructed mice expressing human TDP-43-A315T under control of the mouse prion protein $(\mathrm{PrP})$ promoter. This mutation was found in a St. Louis family with familial ALS. The animals made human protein in amounts approximately three times that of endogenous mouse TDP-43, and expressed the transgene most highly in the brain and spinal cord. The mice first showed motor symptoms at three to four months of age, and after four and a half months they were unable to support their own body weight, using their limbs to scoot around on their stomachs. Survival averaged 154 days, although it varied between approximately 100 days and up to 240. Necropsies of end-stage animals confirmed degeneration of motor axons, and mutant animals at end-stage possessed only 80 percent as many motor neurons as wild-type control mice. The TDP43 mouse has more upper motor neuron involvement than the SOD1 mouse, where symptoms are primarily caused by degeneration of lower motor neurons, Baloh said.

TDP-43 is normally a nuclear protein. Although its exact function is poorly understood, it appears to be involved in several aspects of RNA regulation [2]. In both ALS and FTLD-U, TDP-43 exits the nucleus. Cleaved, hyperphosphorylated, and ubiquitinated, it forms inclusions in the cytoplasm [3]. Researchers have discovered several different TDP-43 mutations in people with ALS [4-7].

In the current study, histopathology of end-stage mice showed ubiquitinated aggregates in both spinal 
motor neurons as well as neurons in the frontal cortex, making the pathology reminiscent of both ALS, where motor neurons are afflicted, and FTLD-U, which affects the frontal temporal lobe. As in humans, the mutant TDP-43 cleared the nucleus of brain cells. However, unlike in human disease, it did not show up in cytoplasmic aggregates. The ubiquitinated inclusions also did not stain positive for $\alpha$-synuclein or tau protein, other common aggregate components. Ronald Klein of the Louisiana State University Health Sciences Center in Shreveport speculated that further examination might still yield a TDP-43 presence: "Perhaps an unknown and so far undetectable TDP-43 fragment conformation was present in the ubiquitin aggregates," he wrote in an e-mail to ARF.

The lack of TDP-43 inclusions could be a sign that the mouse is an inadequate model for human TDP-43 proteinopathy. Alternatively, it could be a clue that nuclear clearing of TDP-43 is the key problem. "Maybe that is where all the business is really at, and these other pathologies are muddying the waters," said Brian Kraemer of the University of Washington in Seattle, who was not involved in the current study. A crucial next step, Baloh said, will be to take a second look at human TDP-43 proteinopathy tissues to see if ubiquitinated inclusions always have TDP-43, or whether some aggregates are TDP-43-negative.

Previous work has shown that caspase- 3 slices TDP43 into 25- and 35-kilodalton carboxyl-terminal fragments [8], and that those fragments are toxic in cell culture [9]. In the mouse model, Baloh and colleagues found that carboxyl-terminal fragments 25 and 35 kilodaltons in size appeared in brain and spinal cord lysates between one and two months of age, preceding noticeable motor symptoms, loss of nuclear TDP-43, and the majority of the ubiquitinated aggregations. The fragments could play a direct role in causing neurodegeneration, the authors suggested.

The TDP-43-A315T mouse is the latest in a diverse line of TDP-43 models. Researchers have studied TDP43 in systems ranging from yeast [10] and mammalian cell culture [11] to nematodes, fruit flies, and zebrafish. In mammals, researchers have used viral vectors to deliver the human TDP-43 gene into the substantia nigra of rats; the animals recapitulated some features of human disease [12].

Adding a transgenic mouse to that panel has been difficult. "For those in the field, it is clear that generating these mouse models is a mammoth task on its own," wrote Samir Kumar-Singh of VIB, University of Antwerp, Belgium, who also was not part of the PNAS publication, in an e-mail to ARF (see full comment below). TDP-43 is essential for development, and knockouts are not viable. Many founder mice fail to reproduce, or the phenotype weakens in successive generations. "I think TDP-43 is just very toxic," Kraemer said. "It is always a tradeoff between getting a mouse that will live and getting a mouse that will express your toxic protein."

Because of these factors, Baloh and colleagues were unsuccessful in engineering a wild-type counterpart to their mutant transgenic line. That leaves open questions of whether its phenotype comes from the specific mutation or simply from too much TDP-43. "It does not negate the findings, but it definitely clouds the interpretation," Baloh said. In the rat model, overexpression of wild-type TDP-43 did cause neurodegeneration [12].

Having a TDP-43 mutant to go along with the SOD1 mouse will strengthen translational research, Baloh suggested: "Testing in two different mouse models might give us a better chance of predicting positive correlations of drugs that work in humans." Medicines that work in both kinds of mice, he said, should be first in line for clinical trials.

\section{RESEARCH BRIEF: THERE IS A FLY IN MY TDP-43 RESEARCH}

A fruit fly is joining the menagerie of TDP-43 animal models, bringing the power of Drosophila genetics to the laboratory bench. Researchers from the Northwestern University School of Medicine in Chicago, Illinois, present the model in a recent paper in PNAS [13]. The flies express wild-type human TDP-43, a protein linked to both ALS and frontotemporal dementia. When the human protein is expressed in motor neurons, the animals show axonal swelling, reduced axonal branching, and ultimately motor neuron loss and mobility problems.

This fly joins a mouse [1], a rat [12], and a host of other TDP-43 animals helping to move TDP-43 research forward.

The current work was led by first author Yan Li and principal investigator Jane Wu. They generated flies expressing TDP-43, hitched to red fluorescent protein (RFP), under neuron-specific promoters. When they expressed the transgene in motor neurons, the flies failed to hatch from the final pupa stage, so they studied the larvae. These moved less than their control counterparts expressing only RFP. Additionally, the researchers created viable adults by turning on the trans- 
gene only in adult tissues, allowing the larvae to develop normally. The adult flies also spent less time in motion than did control flies.

TDP-43 localization appears to be key to its pathogenesis, with the normally nuclear protein migrating into the cytoplasm in disease conditions ([14]; Jane $\mathrm{Wu}$ is a coauthor on this recent paper). Under the microscope, the researchers saw that most of the TDP43 transgene remained nuclear, but when it moved into the cytoplasm the cells displayed swelling, nuclear fragmentation, and cell death.

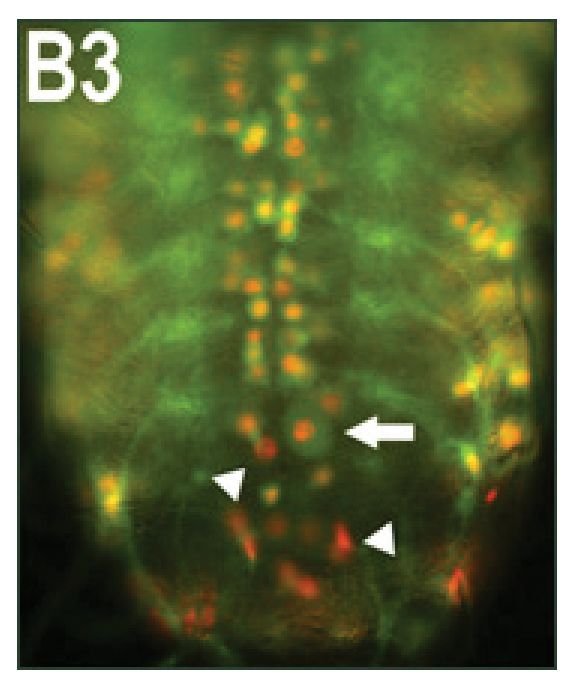

Fig. 1. Transgenic Drosophila expressing human TDP-43 (red) in their motor neurons (membranes in green) evince swelling (arrow) and fragmented or condensed nuclei (arrowheads). Image credit: Proceedings of the National Academy of Sciences USA

TDP-43 contains two amino-terminal RNA-binding domains and a carboxyl-terminal glycine-rich region. Both have been linked to toxicity $[9,15]$. When Li and colleagues expressed solely the carboxyl-terminal fragment in their flies, they found no neurotoxicity, supporting evidence that the amino terminus contributes to pathogenesis.

Many TDP-43 mutations have been linked to disease; however, TDP-43 proteinopathies also exist in people without mutations. In these flies, surplus wildtype TDP-43 was sufficient to cause problems. If that is so in human disease, then reducing TDP-43 might alleviate the symptoms. The researchers created double mutants carrying the human TDP-43 transgene but lacking Drosophila TDP. These animals moved around more than the animals with excess TDP, suggesting that simply increasing TDP levels is enough to cause pathology.
The authors suggest their model will be useful to identify genes that interact with TDP-43 and to test drugs that might dampen the protein's toxic effects.

\section{GOING WILD ABOUT THE LATEST TDP-43 MOUSE MODELS}

Rodents and fruit flies and worms, oh my! The new animal models for TDP-43 proteinopathies, a series of related neurodegenerative diseases, are coming out at a fast and furious rate. The latest to debut are mouse models from researchers at the VIB-University of Antwerp in Belgium [16]. The scientists report a set of mice that express wild-type, human TDP-43 in neurons. The work was led by first author Hans Wils and senior authors Christine Van Broeckhoven and Samir Kumar-Singh in Van Broeckhoven's laboratory.

"This is the first mouse model that has TDP-43 inclusions," Kumar-Singh said. "This is going to be a very powerful series of animal models that can be used for eventual drug targeting." The mice are also the first wild-type TDP-43 overexpression strains to be published; last October Robert Baloh and colleagues at Washington University in St. Louis, Missouri, reported on a mouse carrying human TDP-43 with the diseaselinked mutation A315T [1]. Other animal models under development include rats, zebrafish, fruit flies, and nematodes. "The avalanche has begun," quipped Brian Kraemer of the University of Washington in Seattle. Kraemer was not involved with the current study but has his own TDP-43 knockout mouse in the works. The fish, flies, and worms offer powerful genetic platforms to explore the normal functions of TDP-43; the rodents, it is hoped, will provide systems more akin to human biology.

In people, TDP-43 proteinopathies encompass a spectrum of diseases including some forms of ALS and frontotemporal lobar degeneration (FTLD). Researchers have discovered several TDP-43 mutations in some people with ALS, but in most cases of TDP-43 proteinopathy, it is the wild-type protein that aggregates and is linked to neurodegeneration. The new mouse models may best mimic these sporadic cases, KumarSingh said. In that way it parallels a Drosophila model published recently, which also showed neurodegeneration from the wild-type protein. Ronald Klein and colleagues at the Louisiana State University Health Sciences Center in Shreveport also showed that the wildtype protein could be destructive in rodents. They reported last year on a rat model, produced by injecting 
a viral vector carrying the wild-type TDP-43 gene into the substantia nigra [12].

Wils and colleagues created transgenic mice expressing human TDP-43 driven by the neuron-specific mouse Thy-1 promoter, which activates approximately one week after birth. They used multiple mouse lines to analyze the animals' response to different "doses" of human TDP-43. Of the lines they initially made, the two highest expressers made 1.9- and 2.8-fold more TDP-43 than non-transgenic mice, according to immunocytochemistry analyses. The researchers then self-crossed each line to create homozygotes that expressed approximately double that again, 3.8- and 5.1fold more TDP-43 than non-transgenics. (At the RNA level, quantitative PCR analysis showed that the animals had 0.6- to 1.2-fold of the human TDP-43 gene, compared to the endogenous mouse gene.)

Mice with the greatest TDP-43 load developed their first symptom, abnormal hindlimb reflexes, by just two weeks of age. Symptoms progressed to include stumbling, impaired performance on the rotarod test, and facial muscle spasms. These animals died within a month of birth. The mice expressing 3.8-fold normal TDP-43 evinced hindlimb symptoms at two months, and survived for nearly seven months. The 2.8-fold animals had normal reflexes until 14 months of age. Kumar-Singh and colleagues euthanized these animals before determining their survival rate, but in more recent studies, some of them have survived to 20 months or more, he told ARF in an e-mail. The researchers have not noticed any symptoms in the lowest, 1.9-fold expressers, which they have followed for 19 months. Degeneration of motor neurons, characteristic of ALS, and cortical neurons, as in FTLD, was similarly dose dependent.

TDP-43 appears to be a Goldilocks protein: too much or too little is lethal; a certain amount is just right. Other researchers attempting to make TDP-43 mice have found the project challenging due to the narrow window between getting no phenotype and getting a lethal one. "It tells me that TDP-43 is very well controlled," Kumar-Singh said. Baloh noted that using the Thy-1 promoter as Wils and colleagues did is a promising approach because the transgene is not activated until after birth, so the excess TDP-43 does not interfere with early development.

How might these models relate to the human disease? There is no evidence that people with TDP-43 proteinopathy have abnormally high levels of the protein, but Kumar-Singh theorized that just a little extra TDP-43, over the course of a lifetime, could make neurons vulnerable. Similarly, he noted, increased levels of amyloid- $\beta$ protein precursor - due to duplication or trisomy - can increase the protein's dose and cause Alzheimer's disease. Because levels of the protein are so tightly controlled, simple gene duplication is unlikely to explain TDP-43 proteinopathies. However, Kumar-Singh suggested that polymorphisms in the untranslated regions flanking the TDP-43 gene, or in a gene that influences its expression, might cause increased production. Researchers have already found a variant in the TDP-43 untranslated region in a person who had FTLD [17].

\section{MOUSE VS. MOUSE}

The TDP-43 field now has both wild-type TDP-43 and mutant mouse models, but "I would not say they are a matched pair," Baloh said. They are closer than apples and oranges, more like "oranges and tangerines," he said. Baloh and his colleagues used the mouse prion promoter PrP, which activates expression in glia as well as neurons. They saw TDP-43 levels approximately three times that of endogenous protein, although these data are not directly comparable to the Wils data because they are based on lysate analysis, not immunocytochemistry. But like Kumar-Singh's, Baloh's animals were ill. They suffered motor symptoms by three or four months and lived for an average of five months.

Although human TDP-43 is present throughout the brain in models from both groups, certain neuronal populations were particularly sensitive to the transgene. Layer V cortical neurons, including the primary motor cortex, as well as spinal motor neurons, were hard hit in both models. By confirming Baloh's observations, the new publication shows that the effects on the cortex were not specific to one line. "That is really exciting," Baloh said. "What is it about those neurons that are so vulnerable to TDP-43 toxicity?"

A key hallmark of TDP-43 proteinopathy is aggregates containing ubiquitin and phosphorylated TDP43. The Baloh group observed aggregates, but the accumulations did not contain TDP-43. Van Broeckhoven and colleagues, in contrast, observed that nuclear aggregates, and some cytoplasmic aggregates, in their highest-expressing mice were positive for phosphorylated TDP-43 and ubiquitin. The difference may not be major, Baloh suggested. He noted that the Van Broeckhoven mice were much sicker than his at the time they were sacrificed for immunostaining. The two groups also used different antibodies, and Baloh thinks 
that with the right antibodies, he, too, might see the occasional TDP-43-positive aggregate in his mice.

In TDP-43 proteinopathy, the normally nuclear protein tends to abandon the nucleus for the cytoplasm. Baloh and colleagues noted occasional, but not widespread, nuclear clearing of TDP-43. The KumarSingh group noticed TDP-43 inclusions in both the nucleus and cytoplasm. Caspase-based cleavage of TDP43 , releasing a $25-\mathrm{kDa}$ carboxyl-terminal fragment, has also been linked to the protein's toxicity [9]. Both wild-type and mutant TDP-43 mice showed evidence of these damaging fragments as well. The Van Broeckhoven group compared nuclear and cytoplasmic fractions and discovered the fragments were primarily nuclear in their mice, an unexpected finding given the usual association between cytoplasmic localization and cytotoxicity.

"This is the best TDP-43 animal model to date, because it is a mouse and wild-type TDP-43," Klein wrote of Kumar Singh's work in an e-mail to ARF. This will certainly not be the last model, but with just KumarSingh's and Baloh's work to go on, the question remains: Did the A315T mutation in Baloh's model cause disease, or are those mice sick simply because of overexpression of TDP-43 itself, as in Kumar-Singh's strain? To solve that conundrum, Baloh said, will require a matched pair of mice with wild-type or mutant TDP-43, under the same promoter, at the same level of expression. Kumar-Singh reported that the group has an as-yet-unpublished mutant TDP-43 line that is similar to Baloh's strain.

"There is never a single mouse that will do everything," said John Trojanowski of the University of Pennsylvania in Philadelphia. "It is inevitably the case that you need more than one transgenic mouse to model a disease." His laboratory is pursuing its own mouse model, with a different strategy. Their results are different from, but complementary to, the published mouse models, Trojanowski told ARF.

A single species cannot do everything, either, Kraemer noted. Mice will be useful for the final tests of promising therapeutics, but to find those potential treatments, researchers will need to perform highthroughput screens in vitro or with smaller, cheaper animals - such as worms, flies, or fish.

"The optimistic thing for patients and families is that the pace of research is going so much more rapidly now," Trojanowski said. In comparison, he noted, tau was discovered to be a component of tangles in the mid-1980s, but mouse models were not available until the end of the 1990s. TDP-43 was linked to disease in 2006 [3], and four years later scientists can pick and choose from a plethora of model systems.

\section{REFERENCES}

[1] Wegorzewska I, Bell S, Cairns NJ, Miller TM, Baloh RH (2009) TDP-43 mutant transgenic mice develop features of ALS and frontotemporal lobar degeneration. Proc Natl Acad Sci U S A 106, 18809-18814.

[2] Geser F, Martinez-Lage M, Kwong LK, Lee VM, Trojanowski JQ (2009) Amyotrophic lateral sclerosis, frontotemporal dementia and beyond: the TDP-43 diseases. J Neurol 256, 1205-1214.

[3] Neumann M, Sampathu DM, Kwong LK, Truax AC, Micsenyi MC, Chou TT, Bruce J, Schuck T, Grossman M, Clark CM, McCluskey LF, Miller BL, Masliah E, Mackenzie IR, Feldman H, Feiden W, Kretzschmar HA, Trojanowski JQ, Lee VM (2006) Ubiquitinated TDP-43 in frontotemporal lobar degeneration and amyotrophic lateral sclerosis. Science 314, 130133.

[4] Gitcho MA, Baloh RH, Chakraverty S, Mayo K, Norton JB, Levitch D, Hatanpaa KJ, White CL, Bigio EH, Caselli R, Baker M, Al-Lozi MT, Morris JC, Pestronk A, Rademakers R, Goate AM, Cairns NJ (2008) TDP-43 A315T mutation in familial motor neuron disease. Ann Neurol 63, 535-538.

[5] Sreedharan J, Blair IP, Tripathi VB, Hu X, Vance C, Rogelj B, Ackerley S, Durnall JC, Williams KL, Buratti E, Baralle F, de Belleroche J, Mitchell JD, Leigh PN, Al-Chalabi A, Miller CC, Nicholson G, Shaw CE (2008) TDP-43 mutations in familial and sporadic amyotrophic lateral sclerosis. Science 319, 1668-1672.

[6] Van Deerlin VM, Leverenz JB, Bekris LM, Bird TD, Yuan W, Elman LB, Clay D, Wood EM, Chen-Plotkin AS, MartinezLage M, Steinbart E, McCluskey L, Grossman M, Neumann M, Wu IL, Yang WS, Kalb R, Galasko DR, Montine TJ, Trojanowski JQ, Lee VM, Schellenberg GD, Yu CE (2008) TARDBP mutations in amyotrophic lateral sclerosis with TDP-43 neuropathology: a genetic and histopathological analysis. Lancet Neurol 7, 409-416.

[7] Kabashi E, Valdmanis PN, Dion P, Spiegelman D, McConkey BJ, Vande Velde C, Bouchard JP, Lacomblez L, Pochigaeva K, Salachas F, Pradat PF, Camu W, Meininger V, Dupre N, Rouleau GA (2008) TARDBP mutations in individuals with sporadic and familial amyotrophic lateral sclerosis. Nat Genet 40, 572-574.

[8] Zhang YJ, Xu YF, Dickey CA, Buratti E, Baralle F, Bailey R, Pickering-Brown S, Dickson D, Petrucelli L (2007) Progranulin mediates caspase-dependent cleavage of TAR DNA binding protein-43. J Neurosci 27, 10530-10534.

[9] Zhang YJ, Xu YF, Cook C, Gendron TF, Roettges P, Link CD, Lin WL, Tong J, Castanedes-Casey M, Ash P, Gass J, Rangachari V, Buratti E, Baralle F, Golde TE, Dickson DW, Petrucelli L (2009) Aberrant cleavage of TDP-43 enhances aggregation and cellular toxicity. Proc Natl Acad Sci U S A 106, 7607-7612.

[10] Johnson BS, McCaffery JM, Lindquist S, Gitler AD (2008) A yeast TDP-43 proteinopathy model: Exploring the molecular determinants of TDP-43 aggregation and cellular toxicity. Proc Natl Acad Sci U S A 105, 6439-6444.

[11] Winton MJ, Igaz LM, Wong MM, Kwong LK, Trojanowski JQ, Lee VM (2008) Disturbance of nuclear and cytoplasmic Tar DNA binding protein (TDP-43) induces disease-like redistribution, sequestration and aggregate formation. J Biol Chem 283, 13302-13309.

[12] Tatom JB, Wang DB, Dayton RD, Skalli O, Hutton ML, Dickson DW, Klein RL (2009) Mimicking aspects of frontotem- 
poral lobar degeneration and Lou Gehrig's disease in rats via TDP-43 overexpression. Mol Ther 17, 607-613.

[13] Li Y, Ray P, Rao EJ, Shi C, Guo W, Chen X, Woodruff EA III, Fushimi K, Wu JY (2010) A Drosophila model for TDP-43 proteinopathy. Proc Natl Acad Sci U S A 107, 3169-3174.

[14] Barmada SJ, Skibinski G, Korb E, Rao EJ, Wu JY, Finkbeiner S (2010) Cytoplasmic mislocalization of TDP-43 is toxic to neurons and enhanced by a mutation associated with familial amyotrophic lateral sclerosis. J Neurosci 30, 639-649.

[15] Seyfried NT, Gozal YM, Dammer EB, Xia Q, Duong DM, Cheng D, Lah JJ, Levey AI, Peng J (2010) Multiplex SILAC analysis of a cellular TDP-43 proteinopathy model reveals protein inclusions associated with SUMOylation and diverse
polyUb chains. Mol Cell Proteomics 9, 705-718.

[16] Wils H, Kleinberger G, Janssens J, Pereson S, Joris G, Cuijt I, Smits V, Ceuterick-de Groote C, Van Broeckhoven C, KumarSingh S (2010) TDP-43 transgenic mice develop spastic paralysis and neuronal inclusions characteristic of ALS and frototemporal lobar degeneration. Proc Natl Acad Sci U S A 107, 3858-3863.

[17] Gitcho MA, Bigio EH, Mishra M, Johnson N, Weintraub S, Mesulam M, Rademakers R, Chakraverty S, Cruchaga C, Morris JC, Goate AM, Cairns NJ (2009) TARDBP 3'-UTR variant in autopsy-confirmed frontotemporal lobar degeneration with TDP-43 proteinopathy. Acta Neuropathol 118, 633-645. 